\title{
Intrinsic Transport Theorem
}

\author{
R. M. Kiehn and J. F. Pierce \\ The University of Houston, Houston Texas \\ (Received 21 Oct 1968, final manuscript received 1 April 1969). \\ The Physics of Fluids Volume 12, Number 9, September, 1969
}

August 23, 2001

\begin{abstract}
Cartan's method of exterior differential forms is used to generate an intrinsic transport theorem for continuous media. [with notes updating the original article as of 11/15/2000]
\end{abstract}

\section{Introduction}

In order to demonstrate partially the power of Cartan's ideas, the theory of exterior differential forms [1] will be utilized to develop a set of theorems applicable to continuous media, including an intrinsic transport theorem. As an example, the methods will be applied to the problems of electrodynamics of continuous media, for which it will be demonstrated that the intrinsic transport theorem serves as a first integral for the system of inhomogeneous partial differential equations known as the conservation laws.

Cartan's methods may be used to formulate physical statements in a manner independent of the representation, dimension, and topological nature of the underlying manifold. Since these notions are the ultimate extension of that basic notion of general relativity which is independent from a frame of reference, the application of Cartan's methods demands exposition. Unfortunately the math-

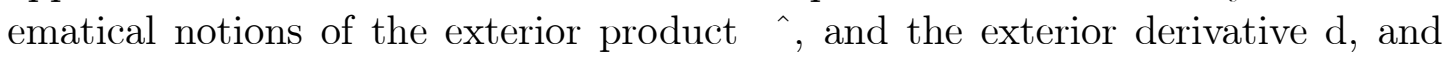
the star operator *, which are the basic tools in the theory of differential forms, have not extensively found their way into the English literature of physics. For 
purposes of definition, the exterior, or hook, product is an associative, distributive pairing of $\mathrm{p}$-forms, which combines 1 -forms, $\alpha$ and $\beta$, according to the rules

$$
\alpha^{\wedge} \beta=-\beta^{\wedge} \alpha ; \alpha^{\wedge} \alpha=0 .
$$

The exterior derivative $d$ is operation which carries a $\mathrm{p}$-form into a $\mathrm{p}+1$ form in a unique manner,

$$
d\left(A_{H} d x^{H}\right)=\left(\partial A_{H} / \partial x^{\mu}\right) d x^{\mu \wedge} d x^{H},
$$

and obeys a modified Leibniz rule when applied to the exterior product of a p-form $\lambda$ and a q-form $\mu$ :

$$
d\left(\lambda^{\wedge} \mu\right)=d(\lambda){ }^{\wedge} \mu+(-1)^{p} \lambda \wedge d \mu .
$$

The star, or Hodge dual, operator $*$ depends upon the metric and is a unique map of a p-form into an N-p form, such that the hook product of the p-form and its dual, yields a scalar tensor density. For the p-form, $\omega^{(p)}=A_{H} d x^{H}$, with $H$ a collective index equal to the set of numbers $\left\{i_{1} \ldots i_{p}\right\}, * \omega^{(p)}$ is equal to $g^{1 / 2} A^{H} d x^{N-H}$, such that

$$
\omega^{(p) \wedge} * \omega^{(p)}=g^{1 / 2} A^{H} A_{H}\left(d x^{1 \wedge} \ldots{ }^{\wedge} d x^{N}\right) .
$$

\section{Theorems}

For purposes of this note it is postulated that the presence of a physical system is specified by a set of metric coefficients $g_{\mu \nu}$, and a covariant vector field whose components are the coefficients of the fundamental 1-form, $\mathcal{A}$. From the theory of exterior differential forms, and in terms of the basic operations defined above, it is possible to prove a number of theorems:

Theorem 2.1. In a space of $N \geq 2$ dimensions, the Hodge dual form, $* A$, satisfies the necessary conditions of integrability; i.e., $(* A)^{\wedge}(d * A)>0$. Let $\beta$ be the integrating factor, such that the $N-1$ form $\beta * A$, is closed: $d(\beta * A)=0$. [Note added 11/15/2000. Every $N$-1 form admits an integrating factor such that the associated vector field $W$ (defined such that $i(W)\left(d x^{1 \wedge} \ldots \wedge d x^{N}\right)=\beta * A$ ) has a zero divergence over a domain that excludes the poles of the integrating factor. It is now known that the Holder norm can be used to construct such an integrating factor.] 
Theorem 2.2. From the 1-form $A$, there may derived by exterior differentiation a 2-form of field intensities, $F=d A$, which by Poincare's lemma is closed: $d F=d d A=0$. This statement establishes the equations for the field intensities [Note added 11/15/2000. For a electromagnetic representation, the PDE's generated by Poincare's lemma are known as the Maxwell-Faraday induction equations. However, the statements are valid for other representations of the 1-form, A, as well. That is, there is an equivalent Maxwell - Faraday induction law for every physical system that can be described by a 1-form of Action. An example is a space time fluid with vorticity or acceleration, for which $d A \neq 0$.]

Theorem 2.3. The Hodge dual of $F$ satisfies the necessary conditions for integrability in a space of $N \quad 4$ dimensions. The existence of the integrating actor as the product of two factors $y f$ is assumed. It follows that the $\mathrm{N}-2$ form of field excitations may be defined by the equation $H=y * F$, which effectively establishes a constitutive link between the field intensities and the field excitations, with the constitutive coefficients determined to within a factor by the metric coefficients, $g_{\mu \nu}$. [Note added 11/15/2000. The factor $f$ which was recognized in 1968, in more recent terms has been called the axion field. This idea was used to obtain a possible representation for the two form of excitations, $H$, in terms of a metric based constitutive relation. Such a metric based constgraint is not necessary, but was used to investigate a possible interaction between the polarization of the EM field and the gravitational field.]

As $(y f * \mathcal{F})$ is closed, it follows that $d H=J$, which are the fundamental equations of field excitation The current, $J$, is an $N-1$ form derived from the distribution, $f: J=H^{\wedge} d(\ln f)$. [Note added 11/15/2000, the PDE's defined by the equations $d H=J$ are known as the Maxwell-Ampere equations in the electromagnetic representation. The format of the current 3-form is due to the assumption of Hodge duality, and the existence of an integrating factor for $H$. In engineering format, the charge density is $\rho=\mathbf{D} \cdot \operatorname{grad}(\ln f)$ and the current density is $\mathbf{j}=\operatorname{grad}(\ln f) \times \mathbf{H}+\mathbf{D} \partial(\ln f) / \partial t]$

Theorem 2.4. : From Poincare's lemma, $J$ is closed leading to the fundamental equation of continuity, $d J=0$. 
Theorem 2.5. From the forms $A$ and $H$ construct the $N-1$ form, $A^{\wedge} H$, which satisfies the identity:

$$
\Psi=\psi d x^{N}=d\left(A^{\wedge} H\right)-\left(F^{\wedge} H\right)+A^{\wedge} J=0
$$

This statement leads to the differential form of the intrinsic transport theorem. [Note added 11/15/2000, the 3-form $\left(A^{\wedge} H\right)$ is now defined as the "topological" Spin 3-form, and the notation has been changed such that the symbol $G$ is used instead of $H$ for the field excitations. Note that $\Psi$ is an $\mathrm{N}$ form, and $\psi$ is a 0 form.]

For arbitrary p-forms, $\omega^{(p)}$, Stokes theorem, $\int_{(p \operatorname{dim} \text { ensional boundary of } M)} \omega^{(p)}=$ $\int_{(p+1 \text { dimensional } M)} d\left(\omega^{(p)}\right)$, forms a basis of global conservation theorems. Consider the $N-1$ form, $\mathcal{A}^{\wedge} \mathcal{H}$, which by Stokes theorem leads to the integral form of the transport theorem,

$$
\int_{\partial M}\left(A^{\wedge} H\right)=\int_{M} d\left(A^{\wedge} H\right)=\int_{M}\left(F^{\wedge} H\right)-\int_{M}\left(A^{\wedge} J\right) .
$$

Theorem 6: The N-form, $\Psi$, is co-closed, $\delta \Psi=0$; the explicit expression for this statement leads to the well known power theorems, fully equivalent to relations involving the divergence of the classical stress-energy tensor in linear systems with constant metric coefficients. In essence, the function $\Psi$ is a first integral to the inhomogeneous conservation laws. It should be emphasized that the theorems stated above are independent of representation and choice of coordinate system. [Note added 11/15/2000. $\delta \Psi=* d * \Psi=0=\psi$ to within a factor. ]

For purposes of rapid comprehension of the above theory, the representation chosen will be classical electrodynamics [2], for which the abstract results may be verified for a given set of coordinates. Using mks units, an arbitrary $x y z t$ reference frame, and Sommerfeld's notation, [3], the field intensity statement, $d F=0$, is equivalent to the first, or homogeneous Maxwell pair of vector equations, based upon the fundamental 1-form, $A=A_{\mu} d x^{\mu}$. The field excitation statement, $d H=$ $J$, leads to the second, or inhomogeneous, Maxwell pair, with the constitutive tensor explicitly determined in terms of the metric coefficients as:

$$
\chi^{\mu \nu \alpha \beta}=1 / 2 y g^{1 / 2}\left(g^{\mu \alpha} g^{\nu \beta}-g^{\mu \beta} g^{\nu \alpha}\right) .
$$

The intrinsic transport theorem becomes

$$
\psi \equiv(\operatorname{div} \mathbf{M}+\partial l / \partial t)-(\mathbf{B} \cdot \mathbf{H}-\mathbf{D} \cdot \mathbf{E})+(\mathbf{A} \cdot \mathbf{J}-\rho \phi)=0,
$$


which is tentatively interpreted as a statement relating the flux of intrinsic angular momentum, $\mathbf{M}=\mathbf{A} \times \mathbf{H}+\mathbf{D} \phi$, and the time rate of change of the intrinsic angular-momentum density, $l=\mathbf{A} \cdot \mathbf{D}$ [which has the units of joule-sec/(meter $)^{3}$ ] to the Lagrangian properties of the field. In Minkowski notation, the contravariant 4-vector density of intrinsic angular momentum flux, $M^{\mu}=(\mathbf{M}, i c l)$, has a nonzero 4-divergence which couples the transport of intrinsic angular momentum to twice the difference between the kinetic and elastic energy density (the reactive energy density), less the interaction energy density of the field. The intrinsic angular momentum is not equivalent to a mechanical angular momentum, as it is independent from the choice of an origin.

The energy-momentum theorems are obtained by setting the components of the N-dimensional gradient of $\psi$ equal to zero. For example, the time component of the gradient of (3) leads to the well known Poynting theorem,

$$
\operatorname{div}(\mathbf{E} \times \mathbf{H})+\mathbf{H} \partial \mathbf{B} / \partial t+\mathbf{E} \partial \mathbf{D} / \partial t+\mathbf{J} \cdot \mathbf{E}=0 .
$$

The new transport theorem (3) as well as the formulation of the Poynting theorem given in (4), has intrinsic significance since it was derived from the theory of forms. (The intrinsic significance cannot be realized if one simply derives the theorem from manipulation of Maxwell's equations.) Equation (3), as well as equation (4), is both gauge invariant and covariant with respect to all smooth coordinate transformations. [Note added 11/15/2000, the claim of gauge invariance is misleading, but true relative to all smooth coordinate transformations]. The conventional treatment of the Poynting theorem attempts to cast (4) into the form of an equation of continuity with source:

$$
\operatorname{div}(\mathbf{S})+\partial W / \partial t+\mathbf{J} \cdot \mathbf{E}=0 .
$$

where the term $W=1 / 2(\mathbf{B} \cdot \mathbf{H}+\mathbf{D} \cdot \mathbf{E})$ is interpreted as the field energy density. Such a formulation does not have intrinsic properties for it is restricted to systems where the constitutive properties are linear and static. However, it is possible to construct the complete momentum-energy flux theorems from (3) in a format related to (5); the gradient of the fundamental scalar, $\psi$, in tensor notation,

$$
\left.0 \equiv \partial \psi / \partial x^{\sigma}=\partial\left(\partial H^{\mu \nu} A_{\nu}\right) / \partial x^{\mu}-1 / 2 H^{\mu \nu} F_{\mu \nu}+J^{\mu} A_{\mu}\right) / \partial x^{\sigma}
$$

can be rearranged to yield 
$\partial\left(F_{\sigma \lambda} H^{\lambda \mu}+1 / 4 \delta_{\sigma}^{\mu} F_{\lambda \nu} H^{\lambda \nu}\right) / \partial x^{\mu}=F_{\sigma \lambda} J^{\lambda}+1 / 4\left(F_{\lambda \nu} \partial H^{\lambda \nu} / \partial x^{\sigma}-H^{\lambda \nu} \partial F_{\lambda \nu} / \partial x^{\sigma}\right)$,

explicitly demonstrating the fact that $\psi$ is a first integral of the inhomogeneous conservation laws. The first term in (7) is the divergence of the Maxwell stress energy tensor; the second term is the Lorentz force density; and the third term is the correction to the force density for non-linear, non-constant constitutive relations [4] often associated with non-inertial frames of reference. By means of the metrical constitutive linkage, Borochoff [5] has demonstrated that the first and third terms combine to form the covariant derivative of the Maxwell stress energy tensor.

Although the application developed above has been in the area of electrodynamics, it should be emphasized that Cartan's methods are independent of representation, suggesting that the application to other problems of continuous media will follow by an appropriate choice of representation. An attempt is being made to exploit this principle in the study of fluids, guided by the recognition that the study fundamental 1-form, $A$, is related to the differential action per unit source. For fluid systems, the unit source is mass, and the fundamental 1-form becomes related to the velocity field of the fluid. The results of this investigation will appear later. [Note added 11/15/2000, this was first done for a Navier-Stokes fluid in Kiehn, R. M. , (1975b), "Intrinsic hydrodynamics with applications to space-time fluids", Int. J. of Eng. Sci. 13, p. 941. Several other applications were developed over the years and many of the publications can be downloaded from my website.].

This work was supported in part by the National Aeronautics and Space Administration - Manned Spacecraft Center, Grant NGR 44-005-022.

\section{References}

[1] E. Cartan, Lecons sur les Invariants Integraux, (Hermann, Paris, 1922); H. Flanders, Differential Forms (Academic Press Inc., New York, 1963)

[2] R. M. Kiehn, Bull Am. Phys Soc., 12, 198 (1967). For source free systems in four dimensions, see: R. Debever, Coll. de Geom. Diff., Louvain, 217, (1951), and C.W.Misner and J. A. Wheeler, Ann. Phys. (N. Y.) 2, 525 (1957)

[3] A. Sommerfeld, Electrodynamics, (Academic Press Inc., NewYork, 1952) 
[4] The formulation without the usual assumptions on the constitutive relations may be found in C. Moller, The Theory of Relativity, (Clarendon Press, Oxford, England, 1962) p.202, and E. J. Post, The Formal Theory of Electromagnetics (North Holland Publishing Company, Amsterdam, 1962), p 88

[5] R. Borochoff (Private communication) 